\section{Current issues in}

\section{cosmology}

Joseph Silk

Department of Physics, Nuclear and Astrophysics Laboratory

Keble Road, Oxford OX1 3RH, United Kingdom

$T_{\mathrm{o}}^{\mathrm{o}}$ illustrate the status of modern cosmology, I have chosen three issues that have played a central role in cosmology ever since the emergence of the Big Bang theory as the dominant cosmological model.

\section{Age of the Universe}

The issue of the age of the Universe was the focus of much discussion during the $19^{\text {th }}$ century, centring on the geological age of the Earth. A major breakthrough was the discovery of the longevity of the Sun, via thermonuclear energy sources, in the first half of the $20^{\text {th }}$ century, coupled with the realization that the expansion age of the Universe exceeded the age of the Sun and was comparable to the ages of the oldest stars in our Galaxy. The scene had shifted: from the age of the Earth versus that of the Sun to the ages of the oldest stars versus that of the Big Bang.

During the second half of the $20^{\text {th }}$ century, a controversy raged around the value of the Hubble constant, the inverse of which gives a first approximation to the age of the Universe. One school, led by

a controversy raged Gerard de Vaucouleurs, argued vociferously for a high value of Hubaround the value of ble's constant $(100 \mathrm{~km} / \mathrm{Mpc} / \mathrm{sec})^{1}$. If de Vaucouleurs and his supporters had prevailed, the Big Bang would have been in considerable difficulty. However, Allan Sandage, Gustav Tammann and their supporters were equally strong advocates for a low value of Hubble's constant of $50 \mathrm{~km} / \mathrm{Mpc} / \mathrm{sec}$. Only with the passage of time and the entry into the fray of a new generation of astronomers who exploited new instruments, most notably the Hubble Space Telescope, did the situation converge. High precision observations of key distance calibrators such as Cepheid variable stars and supernovae in distant galaxies resulted in a consensus that Hubble's constant is $(70 \pm 7) \mathrm{km} / \mathrm{Mpc} / \mathrm{s}$, a value that poses no problem whatsoever in reconciling the oldest stellar ages with the age of the Universe.

The canonical cosmological model has an age of $14 \mathrm{Gyr}$. The cosmological age is consistent with two independent age determinations for the Milky Way galaxy. Stellar evolution applied to the oldest globular cluster stars gives an age of $13 \mathrm{Gyr}$, and radioactive dating from measuring abundances of $U$ and $T h$ in extremely metal-poor halo stars yield an age of $12 \mathrm{Gyr}$.

There are also cosmological measures of the Hubble constant that are independent of distance. One utilizes the Sunyaev-Zeldovich effect on galaxy clusters to combine X-ray measurements of galaxy clusters with the microwave (Rayleigh Jeans) decrement in the cosmic blackbody background radiation due to traversal across the hot intracluster gas by the microwave photons to give an absolute distance measurement. Yet another approach makes use of the cosmic microwave background radiation anisotropies, where the height of the first acoustic peak is sensitive to the Hubble constant, given a cosmological model.
There is complete consistency between the various Hubble constant determinations. There is no age controversy for the Big Bang.

\section{Galaxy Formation}

Galaxy formation theory is not in a very satisfactory state. This stems ultimately from our lack of any fundamental understanding of star formation. There is no robust theory for the detailed properties of galaxies. In contrast, the evolution of the dark matter, which is by far the predominant constituent of the Universe, is well understood. Hierarchical formation of large-scale structure in a cold dark matter-dominated Friedmann-Lemaître Universe has successfully confronted essentially all observations, ranging from deep surveys of the galaxy distribution and the formation of galaxy clusters to the temperature fluctuations in the cosmic microwave background.

The advent of high-resolution N-body simulations has revealed challenges for galaxy formation. Dark halos contain considerable sub-structure, amounting to circa $10 \%$ of the halo mass, and continuing down to unresolved scales of $106 \mathrm{M} \$$ or smaller. This has led to two problems. One is that the predicted number of dwarf galaxy satellites exceeds that observed around the Milky Way by an order of magnitude. Even very low surface brightness dwarfs are easily observed in our local environment, and efficient mass loss prior to star formation has been invoked to resolve this discrepancy.

A second problem is that, on the one hand, the initial angular momentum of a typical protogalaxy, when the halo first collapsed, can reproduce the observed size distribution of disks, if angular momentum is approximately conserved during baryon infall and disk accretion, but that, on the other hand, the numerical simulations show that most of the angular momentum is actually lost to the dark halo. The clumpiness induces strong angular momentum transfer via tidal torqueing and dynamical friction from the dissipating baryons to the energy-conserving dark matter. The resulting disks have too little specific angular momentum by an order of magnitude.

Another result from the high-resolution simulations of galaxy halos is that the dark halo has a central concentration and a central cusp. This implies for example that about half the mass within the half-light radius (for our Galaxy this corresponds to near the solar circle) is in the form of cold dark matter (CDM). This apparently contradicts bulge microlensing studies for the Milky Way, which permit a CDM fraction of at most $10 \%$ within the solar circle, when combined with the rotation curve and with infrared stellar population modelling derived from the results of the Deep Infrared Background Experiment (DIRBE) that was flown on the Cosmic Background Explorer (COBE). A similar conclusion applies to barred galaxies, for which dynamical studies conclude that self-gravity of the bars, which consist primarily of stars, must dominate the inner gravitational potential. In contrast, a recent study of a non-barred spiral finds that detailed modelling of the rotation curve with a maximal disk requires an axially-symmetric dark-matter contribution of about $30 \%$ within an optical radius.

There is another observational hurdle to overcome. Low surface brightness spirals are everywhere dark-matter dominated, and so provide outstanding laboratories for dark matter studies via rotation curves. High-resolution Ha rotation curves reveal a wide array of central profiles. Most systems have soft cores without any indication of a central cusp.

Cold dark matter is seriously challenged. Whether it is actually dead is quite another matter. Nevertheless, to confront this possi- 


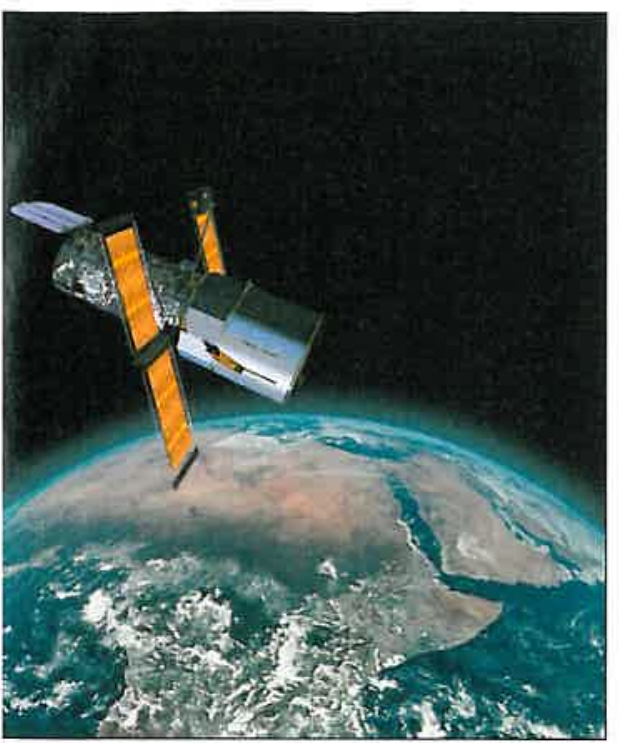

Inly with the passage of time nd the entry into the fray of a Iew generation of astronomers using] new instruments, most Iotably the Hubble Space elescope, did the situation onverge. bility, there have been numerous attempts to resurrect CDM. These come under two distinct guises: tinkering with the particle physics or elaborating on the astrophysics. Modified-particle dark-matter can no doubt be developed to explain all of the required dark matter properties. However the seductive simplicity of SUSY, with the lightest stable supersymmetric particle, the neutralino, as an attractive candidate for CDM, is lost.

An alternative approach is via the astrophysics of galaxy formation. Can the dark matter profile be modified by astrophysical processes? The answer is: perhaps. Supermassive black holes, for better or for worse, are intimately connected with the process of galaxy spheroid formation. Whether they aid and abet formation of the first stars remains a mystery. There are certainly dynamical and most likely astrochemical links. The supermassive black-hole (SMBH) merger results in heating of the dark matter cusp. The dark matter is heated and acquires angular momentum. The result is that the concentration and cusp are likely to be modified. However the angular momentum acquired by the halo will help in reinjecting angular momentum into infalling gas clouds that form the disk over a time-scale of a Gyr or longer.

It remains to be seen whether the ultimate answer lies in the dynamical feedback of SMBH formation and evolution on dark halo cores, or on a new prescription that modifies the physics of $\mathrm{CDM}$, or possibly in fundamental physics whereby on large scales unanticipated changes in our 4-d Einstein gravity may be appearing, such as might be associated with the influence of higher dimensions.

\section{Dark Matter}

The favoured candidate for CDM is the lightest stable SUSY relic particle. This must be neutral (to avoid already having been detected) and its mass is constrained by accelerator searches and theoretical considerations of thermal freeze-out to lie in the range $50 \mathrm{GeV}$ to a few $\mathrm{TeV}$. The relic density is determined when annihilations and pair production go out of thermal equilibrium in the early Universe at $T \approx m_{x} / 20 \mathrm{kK}$, and one infers that the density in relic particles, relative to the critical density of an Einstein-de Sitter Universe, satisfies $\Omega_{x} \propto \sigma_{a n n}{ }^{-1}$, where $\sigma_{a m n}$ is the annihilation cross-section extrapolated to the low temperature limit and $m_{x}$ is the mass of the relic particle. For typical weak interaction values of $\sigma_{a n n}$, one finds that $\Omega_{x} \approx 0.3$ is required to account for the dark matter content of the Universe. Via studying a grid of supersymmetric (SUSY) models, one can infer a range of particle masses from the annihilation cross-section. Were it not for the accelerator bounds on the sparticle (i.e., supersymmetric partner-particle) masses, the uncertainty in $m_{x}$ would span some 5 orders of magnitude.

The annihilation cross-section and particle mass is constrained. So also is the elastic scattering cross-section once the annihilation cross-section is specified. This means that one can now consider possible detection schemes. The obvious one is direct detection by elastic scattering. Use of annual modulation of the incident flux on a terrestrial detector has led to a tentative detection (DAMA) ${ }^{2}$ that requires an implausibly large cross-section given the suite of minimal SUSY models and is marginally inconsistent with another experiment (CDMS) ${ }^{3}$. Annihilations result in hadronic jets that decay into gamma rays, high-energy electron-positron pairs, proton-antiproton pairs and neutrinos, all of which are potentially detectable as galactic halo signals.

Future observations may greatly help in pinning down the CDM characteristics. Annihilations generate high-energy neutrinos and gamma rays that propagate freely in the halo and detection would provide unambiguous support for annihilating Weakly Interacting Massive Particles (WIMPs). The predicted fluxes are within the anticipated sensitivity of the ANTARES, AMANDA (neutrino) and GLAST (gamma ray) detectors ${ }^{4}$, now under construction.

\section{Footnotes}

${ }^{1}$ Distances in astronomy are usually given in parsec $(\mathrm{pc})$;

$1 \mathrm{pc}=3.085 \cdot 10^{16} \mathrm{~m}=3.26$ light year

${ }^{2}$ 'Particle DArk MAtter searches with highly radiopure scintillators at Gran Sasso', $c f$. article by Y. Ramachers.

${ }^{3}$ The 'Cryogenic Dark Matter Search (CDMS)' experiments aim to measure the recoil energy imparted to detector nuclei through neutralino-nucleon collisions by employing sensitive phonon detection equipment coupled to arrays of cryogenic germanium and silicon crystals.

${ }^{4}$ ANTARES is an underwater neutrino telescope project in the Mediterranean Sea (the Astronomy with a Neutrino Telescope and Abyss Environment RESearch'), AMANDA is a neutrino telescope in the South Polar ice cap (the 'Antarctic Muon And Neutrino Detector Array') and GLAST is the 'Gamma Ray Large Area Space Telescope'.

\section{About the author}

Joseph Silk is a 58 year old British astronomer currently working at Oxford University. His scientific career led him to positions in the most renowned universities and he has been awarded numerous prizes. In his research he focuses on cosmology, particularly on cosmos background radiation. He has written or co-written more than 300 specialised scientific essays. He is also the author of many popular works and books on science.

In this overview, Silk wants to make basic notions in cosmology clearer and he tries to explain why we will probably never know whether or not the universe is finite. 